\title{
January 2015 Critical Care Case of the Month: Who's Your Momma?
}

\author{
Carlos Hartmann, MD \\ Layth Al-Jashaami, MD \\ Timothy T. Kuberski, MD \\ Department of Medicine \\ Maricopa Integrated Health Services \\ Phoenix, AZ USA
}

\section{History of Present Illness}

A 39-year-old Hispanic woman was admitted complaining of shortness of breath and bilateral lower extremity edema. She was felt to be in acute hypoxic respiratory failure.

\section{Past Medical History}

- Systemic lupus erythematosus

- Congestive heart failure with an ejection fraction of $40 \%$

- End-stage renal disease on dialysis secondary to lupus nephritis

\section{Medications}

- Rituximab 550 mg once a week

- Prednisone $40 \mathrm{mg}$ daily

- Plaquenil $200 \mathrm{mg}$ twice a day

\section{Physical Examination}

The patient was tachypneic in obvious respiratory distress. She was afebrile. Crackles at the bases. Heart was tachycardic. There was $3+$ bilateral pitting pretibial edema.

\section{Laboratory}

CBC: Hemoglobin $7.8 \mathrm{~g} / \mathrm{dL}$, WBC $11 \times 10^{9}$ cells per liter, differential: neutrophils $98 \%$, eosinophils $0 \%$, lymphocytes $1 \%$.

Electrolytes: Potassium $5.8 \mathrm{mEq} / \mathrm{L}$, sodium $143 \mathrm{mEq} / \mathrm{L}$, creatinine $3.3 \mathrm{~g} / \mathrm{dL}$, BUN 98 $\mathrm{mg} / \mathrm{dL}$.

Brain naturetic peptide: $4055 \mathrm{pg} / \mathrm{ml}$.

\section{Imaging}

Admission chest $x$-ray showed cardiomegaly and bilateral interstitial prominence suggestive of congestive heart failure.

Which of the following are appropriate initial management?

1. Bronchoscopy with bronchoalveolar lavage

2. Hemodialysis

3. Increased methylprednisolone for a potential lupus "flare"

4. 1 and 3

5. All of the above 


\section{Correct! \\ 5. All of the above}

The cause for her respiratory failure is not apparent. The patient is an immunocompromised host and bronchoscopy with bronchoalveolar lavage and empiric antibiotics along with blood and urine cultures are appropriate to diagnose and treat a potentially life-threatening infection. Hemodialysis was appropriate to treat her apparent congestive failure. Immunosuppressive therapy was escalated to $1 \mathrm{~g}$ of methylprednisolone for a possible lupus flare.

Her cultures were negative. Due to respiratory failure she was intubated and placed on a ventilator initially improving clinically and radiographically. After a few days, there was clinical deterioration and worsening chest X-ray (Figure 1).

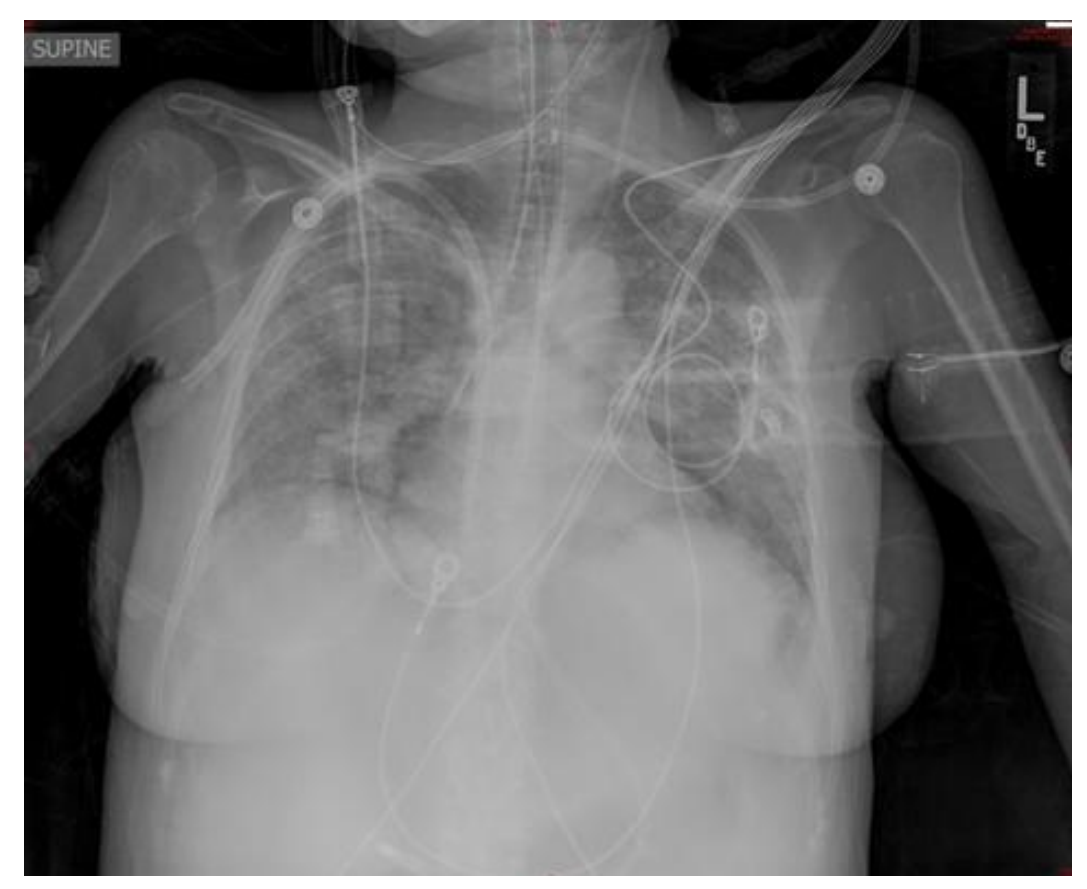

Figure 1. Portable chest x-ray taken two weeks after admission.

She also developed a violaceous, non blanching macular rash on her buttocks and lower extremities with a few hemorrhagic bullae in a dermatomal distribution. She also developed rectal bleeding. An esophagogastroduodenoscopy (EGD) was suggestive of pseudomembranous colitis. She was started on oral vancomycin.

Which of the following is/are appropriate?

1. Acyclovir for possible Herpes zoster

2. Open lung biopsy

3. Repeat bronchoscopy with bronchoalveolar lavage

4. 1 and 3

5. All of the above 


\section{Correct!}

\section{1 and 3}

Again it is unclear why the patient is clinically declining. She is at high risk for Herpes zoster and empiric acyclovir would seem appropriate. Bronchoscopy with

bronchoalveolar lavage is easy and safe to do through an endotracheal tube. Open lung biopsy could be considered if the bronchoalveolar lavage is negative for infection but seems overly aggressive at this time.

A 2-3 $\mathrm{mm}$ organism was seen in the bronchoalveolar lavage fluid (Figure 2).

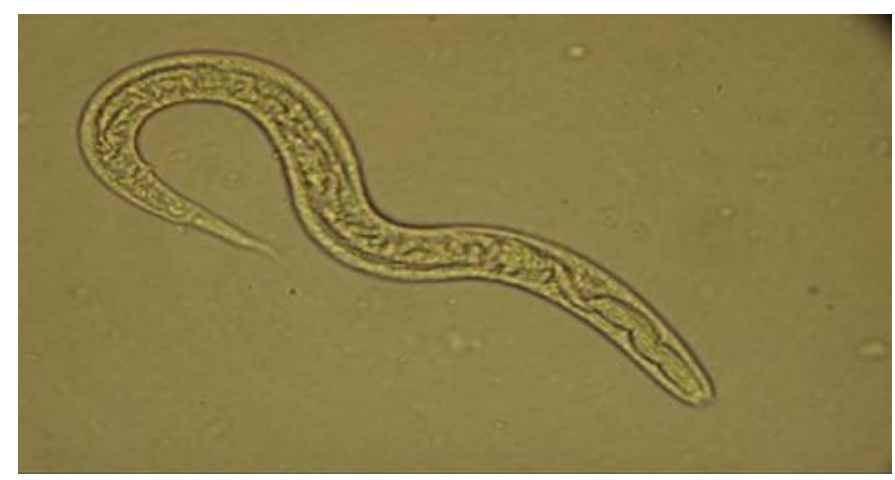

Figure 2. Photomicrograph of the organism seen in the bronchoalveolar lavage fluid.

A smaller but similar organism was seen in the biopsy from the EGD (Figure 3).

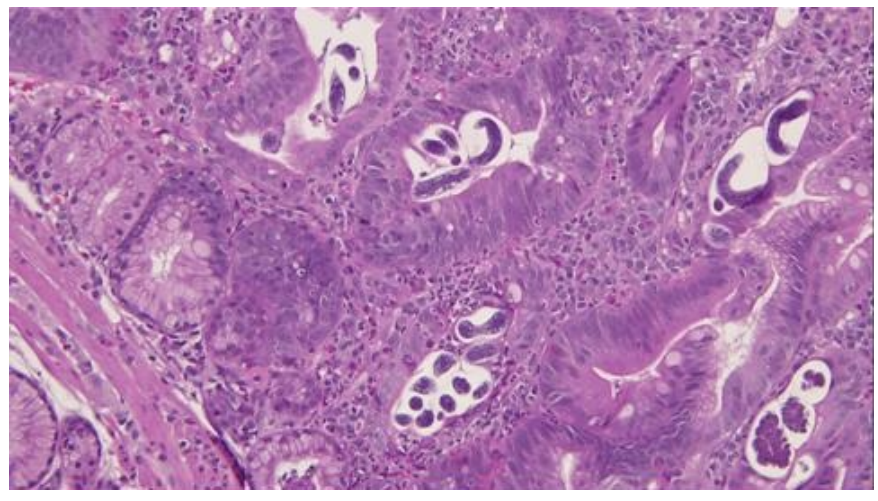

Figure 3. Low power view of the duodenal biopsy (H \& E).

Which of the following drug(s) should be given to the patient?

1. Ivermectin

2. Piperacillin/tazobactam

3. Praziquantel

4. 1 and 3

5. All of the above 


\section{Correct!}

\section{Ivermectin}

The patient is infected with Strongyloides stercoralis, a roundworm commonly known as the thread worm. In conditions of immunodeficiency the worm may disseminate causing a potentially fatal Strongyloides hyperinfection syndrome (SHS) (1). Ivermectin is the drug of choice (2). Thiobendazole is an alternative but less effective. Praziquantel is used to treat tapeworm infections. Piperacillin and tazobactam (Zosyn $®)$ is an antibacterial and has no activity against parasites.

The cycle of Strongyloides is given in Figure 4.

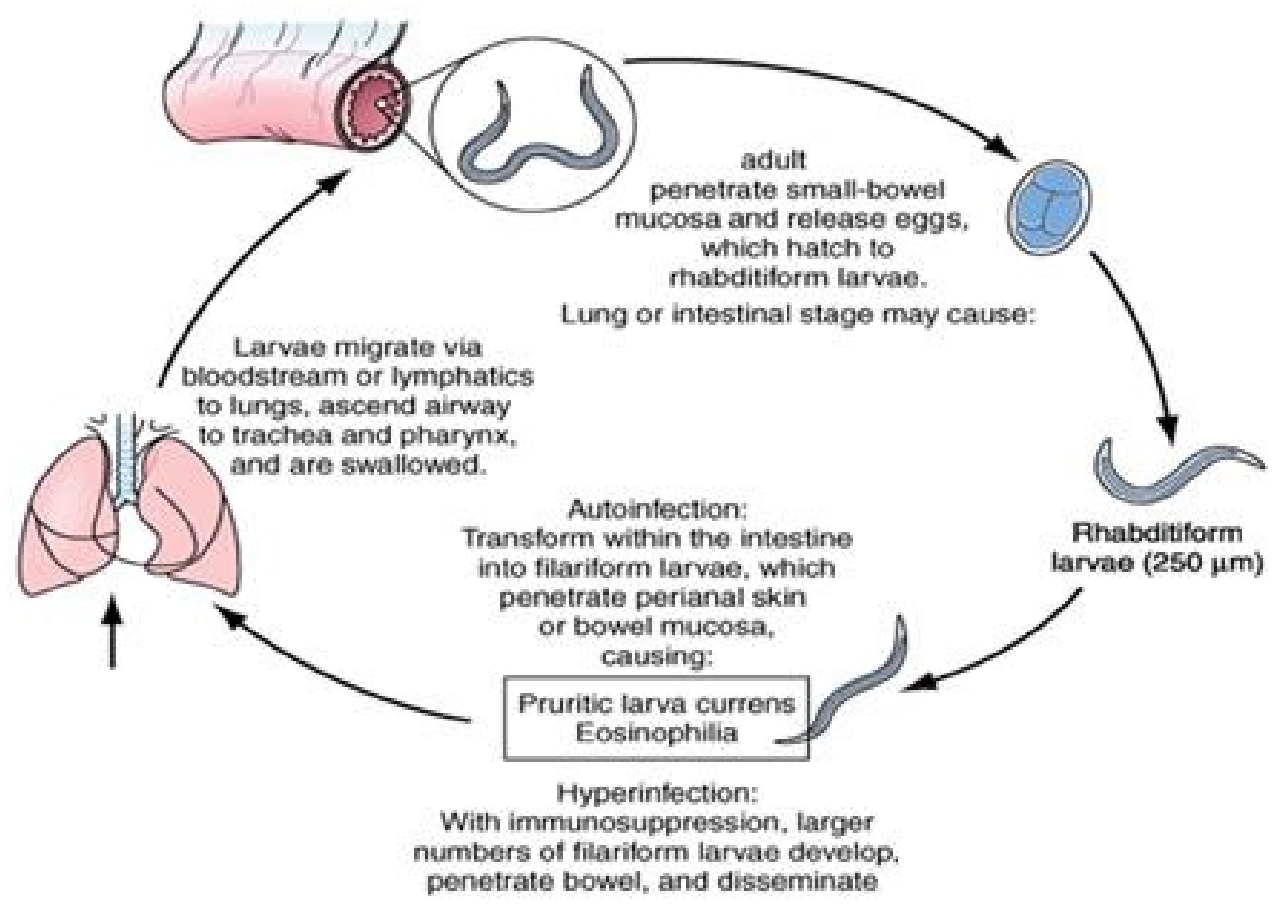

Figure 4. Life cycle of Strongyloides.

Strongyloides larvae exist in a free-living form in contaminated soil and infects humans via skin penetration through shoeless feet. Cutaneous penetration by the parasite produces characteristic skin lesions, called "larva currens". After penetrating the skin the larvae gain access to the venous circulation and are carried to the lungs; then penetrates the alveolar wall. They are then able to migrate to the glottis, where they are swallowed, allowing the nematode access into the gastrointestinal tract, where it resides in the small bowel. The larvae then burrowed into the mucosa and transform into adults and produce eggs. The eggs develop into rhabditiform larvae which are released into the gastrointestinal tract where they can live for decades. However, in the immunocompromised host the rhabditiform larvae are capable of reinfecting its host by invading the bowel wall or perianal skin (3). As in our patient, acceleration of the life cycle, by autoinfection where the rhabditiform larvae within the host resulting Strongyloides hyperinfection syndrome (SHS). 
Strongyloides can be quiescent for many years in an infected host who travel to the United States from endemic areas. Since Strongyloides is rare in the United States, often times diagnosis is not made pre-mortem. Perhaps the largest population at risk is that of patients receiving immunosuppressive therapy for another disease, especially those whose cases are managed with glucocorticoids. As our case illustrates, steroid therapy and its associated suppression of cell-mediated immunity can remove the controls from controlled infection with S. stercoralis and resulting in dissemination. While patients from endemic areas are at obvious risk, there is also a risk to those who travel to endemic areas, including veterans of military service (3).

Our patient was chronically immunosuppressed by steroid therapy which accelerated Strongyloides through autoinfection causing dissemination. Because of the severity of her illness she was treated with both ivermectin and thiobendazole. Survival of disseminated strongyloidiasis is possible, and early diagnosis and treatment improve outcome. Mortality of disseminated strongyloidiasis remains at $43 \%$; it climbs to $77 \%$ with the loss of immune function. Death is frequently the result of either secondary infectious or respiratory failure $(3,4)$. This patient was from Mexico where Strongyloides is endemic and asking "whose your momma" might give a clue to this difficult diagnosis.

\section{References}

1. Simpson WG, Gerhardstein DC, Thompson JR. Disseminated Strongyloides stercoralis infection. South Med J. 1993;86:821-5. [CrossRef] [PubMed]

2. CDC. Parasites - Strongyloides. Available at: http://www.cdc.gov/parasites/strongyloides/health professionals/ (accessed 12/20/14).

3. Igra-Siegman Y, Kapila R, Sen P, Kaminski ZC, Louria DB. Syndrome of hyperinfection with Strongyloides stercoralis. Rev Infect Dis. 1981;3(3):397-407. [CrossRef] [PubMed]

4. Keiser PB, Nutman TB. Strongyloides stercoralis in the immunocompromise population, Clin Microbiol Rev. 2004;17(1):208-17. [CrossRef] [PubMed] 